\title{
Engineered Growth of Organic Crystalline Films Using Liquid Crystal Solvents
}

\author{
F. Scott Wilkinson, Ronald F. Norwood, Joseph M. McLellan, \\ L. Rhys Lawson, David L. Patrick \\ Department of Chemistry, Western Washington University, 516 High St., Bellingham, WA, 98225
}

\section{$\underline{\text { Supplemental Information }}$}

\section{Experimental Conditions and Materials.}

Tetracene vapor was generated by thermal evaporation at $190-230{ }^{\circ} \mathrm{C}$ from a heated graphite crucible placed 6" - 8" below the substrate. Deposition was performed at 1 atm in $\mathrm{N}_{2}$, Ar or air. Atmospheric pressure deposition was apparently possible because small particles that formed in the gas phase dissolved in the LC layer, a process seemingly driven by Ostwald ripening. The largest of these particles were several microns in size, determined by capturing them on adhesive tape placed near the substrate and imaging them with SEM. Atmospheric pressure deposition was required to prevent evaporation of the LC, but also provides two important advantages: (i) the potential for greatly reduced cost of manufacturing compared to high or ultrahigh vacuum, and (ii) potential compatibility with nontraditional vapor generation methods such as electrostatic powder coating, which can be used to prepare truly large area films.

Heating tetracene to $\sim 200{ }^{\circ} \mathrm{C}$ in air results in some oxidation. To test the effects of this, in some experiments the deposition chamber was pumped to $1 \times 10^{-1}$ Torr and purged with $99.999 \%$ Ar up to eight times. The resulting films did not differ significantly from those grown in ambient air. This may be because the oxidation products have a lower vapor pressure and hence do not evaporate significantly at the temperatures used, or it may be due to gettering by the LC solvent, which reduces incorporation of more soluble impurities into the growing crystals. All experiments involving pentacene were performed under inert gas.

Substrates typically measured $1-2 \mathrm{~cm}$ in diameter and were mounted to a temperature-controlled holder. LC, PVA and lecithin were applied by spin coating. Alignment of PVA films was achieved by gentle unidirectional rubbing using a soft cloth.

The effect of increasing coverage was to increase both the size of the largest crystals and, to a certain point, the density of crystals (i.e. number of crystals per unit area). Once crystals covered approximately $1 / 3$ of the projected surface area, nucleation of new crystals slowed, while existing ones continued to grow. As crystals grew in thickness, additional LC pooled around them, keeping them submerged.

\section{Liquid Crystals:}

ZLI-3417-000 is a multicomponent LC mixture used in $2^{\text {nd }}$ minimum twisted nematic displays. It was purchased from Merck KGaA, Darmstadt, Germany, and has a proprietary formulation. Crystallization temperature: $<-20{ }^{\circ} \mathrm{C}$, clearing point: $94{ }^{\circ} \mathrm{C}$.

MBBA (n-4'-methoxybenzylidene-n-butylaniline) is a single component room-temperature nematic LC. It was purchased from Frinton Laboratories, Vineland, NJ. Crystallization temperature: $21{ }^{\circ} \mathrm{C}$, clearing point: $47^{\circ} \mathrm{C}$.

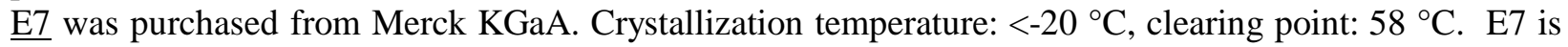
a euctectic nematic LC mixture consisting of four components:

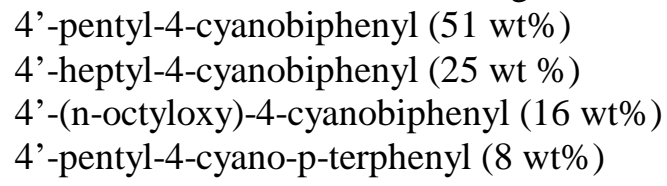


Experimental conditions for specific figures:

2A: $\operatorname{Si}(100)$ substrate coated with mechanically rubbed PVA and the LC ZLI-3417. Substrate temperature $34{ }^{\circ} \mathrm{C}$, deposition performed in air for 2 hours. LC was removed prior to photographing by rinsing in cold hexane.

2B: Si(100) substrate coated with mechanically rubbed PVA and the LC and the LC E7. Substrate temperature $35{ }^{\circ} \mathrm{C}$, deposition performed in air for $45 \mathrm{~min}$. LC was removed prior to photographing by rinsing in cold hexane.

2C: $\mathrm{Si}(100)$ substrate coated with mechanically rubbed PVA and the LC and the LC MBBA. Substrate temperature $34{ }^{\circ} \mathrm{C}$, deposition performed in air for 2 hours. LC was removed prior to photographing by rinsing in cold hexane.

2D: Same conditions as $2 \mathrm{~A}$

2F: Same conditions as 2A, except that the PVA alignment layer was not mechanically rubbed.

2G: Same conditions as $2 \mathrm{~A}$.

2. X-Ray Diffractometry. X-ray diffraction measurements were performed on tetracene films grown using the LC ZLI-3417 in Bragg-Brentano geometry with a Rigaku powder diffractometer (Cu k- $\alpha$ line). Diffractograms agreed with tetracene's previously reported bulk triclinic structure. ${ }^{1}$ The observed reflections occurred exclusively from the (001) family of planes, indicating that crystals oriented with the ab-plane of the unit cell parallel to the substrate.

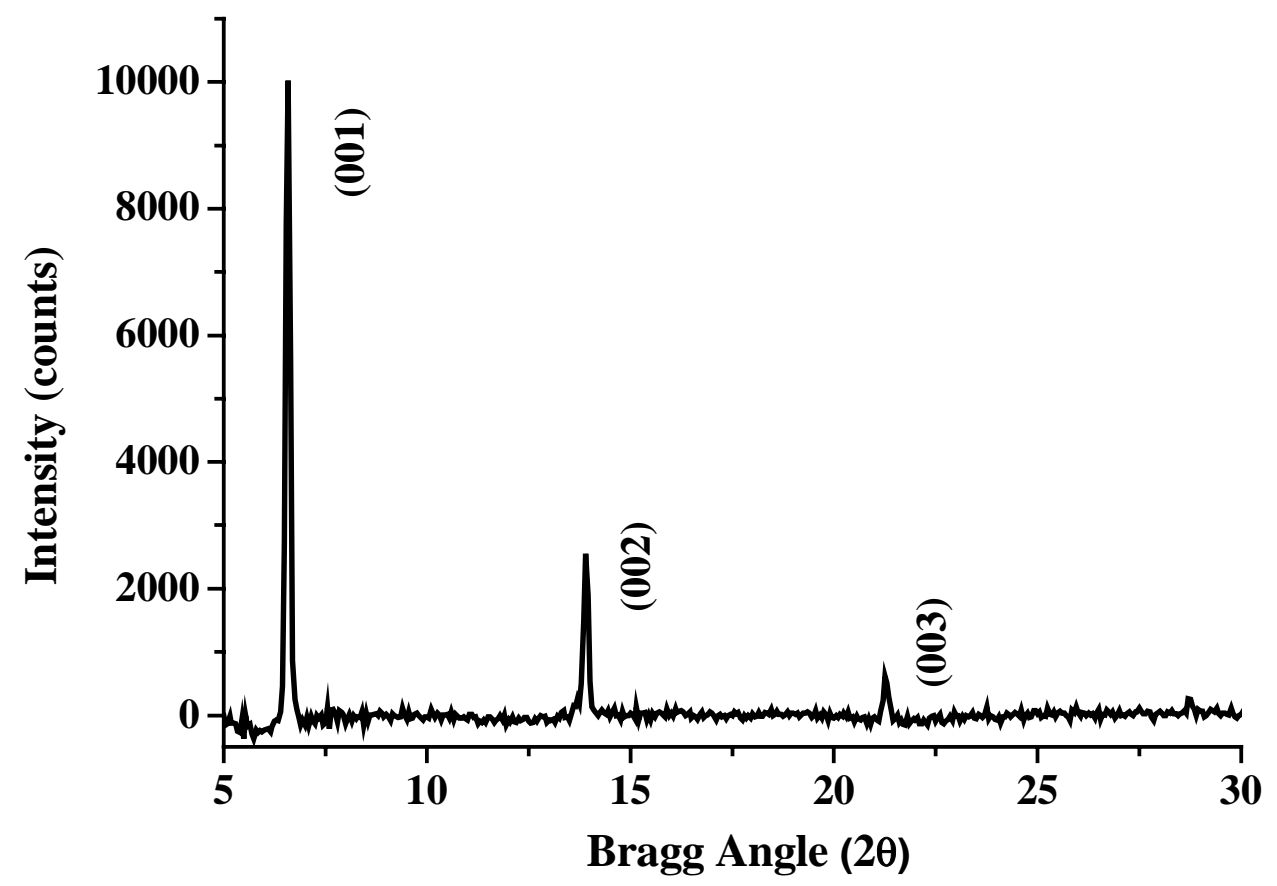

Fig. S1. X-ray diffractogram of tetracene on $\mathrm{SiO}_{2}(100)$ / rubbed PVA / ZLI-3417. 
3. Fluorescence Spectroscopy. The emission spectrum of an oriented tetracene film is shown in Figure 4.2. The sample was illuminated using monochromatic $500 \mathrm{~nm}$ light, with a $515 \mathrm{~nm}$ long-pass filter between the sample and detector. The spectrum reveals a prominent peak at $534.7 \mathrm{~nm}$, corresponding to the (0-0) transition, and a second peak at $557.3 \mathrm{~nm}$ for the (0-1) transition peak. Peak positions were found by curve fitting the spectrum using a two peak Lorentzian model. The spectrum agrees with previous studies of both polycrystalline and single crystal tetracene. ${ }^{2,3,4}$

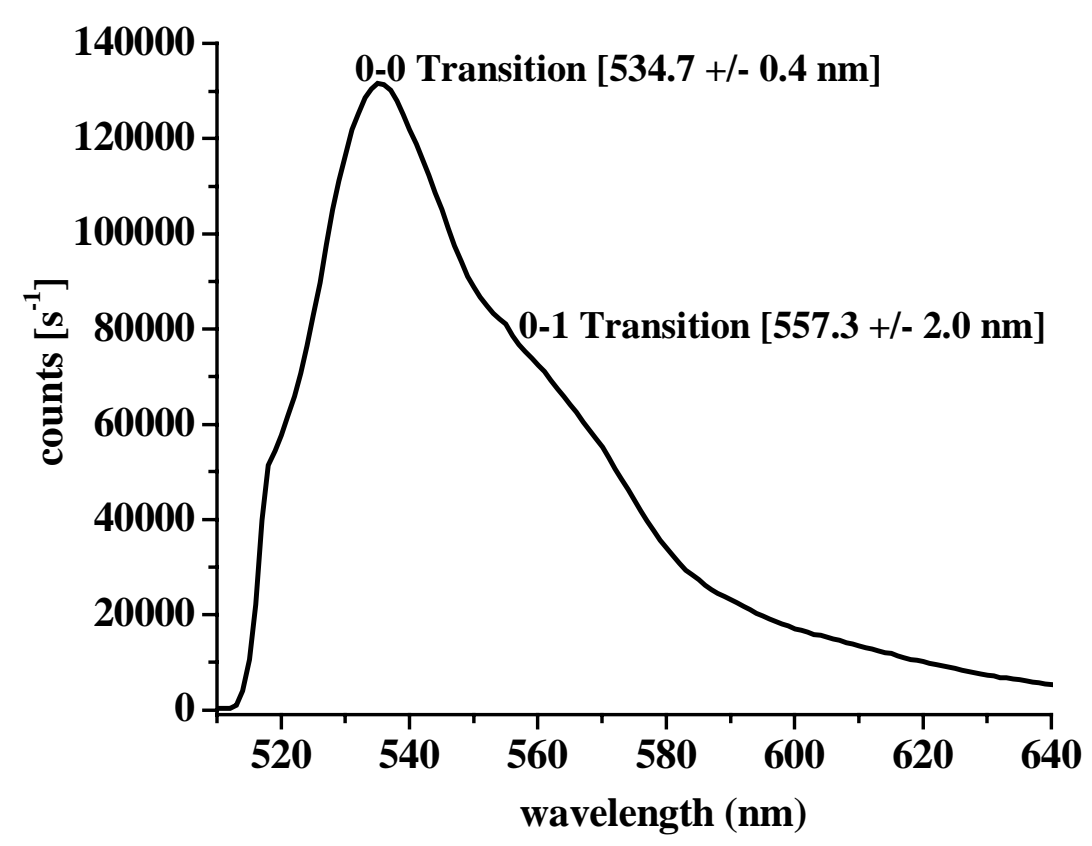

Fig. S2. Fluorescence emission spectrum of tetracene on $\mathrm{SiO}_{2}(100)$ / rubbed PVA / ZLI-3417, with assigned vibronic transitions and their respective wavelengths. 
4. UV-Vis Absorption Spectroscopy. Absorption spectroscopy measurements were performed with samples grown on transparent glass substrates using both polarized an unpolarized light. Peak positions were found to be in good agreement with recent studies of crystalline tetracene. , 6, 7, 8, $9^{2}$

The polarization-dependence of the two lowest energy Davydov components, indicated in Fig. $\mathrm{S} 3$, combined with the finding from x-ray diffraction that the $a b$-plane is parallel to the substrate, can be used to analyze crystallographic orientation. ${ }^{10}$ Although this information is limited to an angular resolution of $\pm 45^{\circ}$, due to the uniaxial symmetry imposed by the LC, the results provide an independent confirmation of the orientational assignment from shape modeling of individual crystals discussed in the paper.

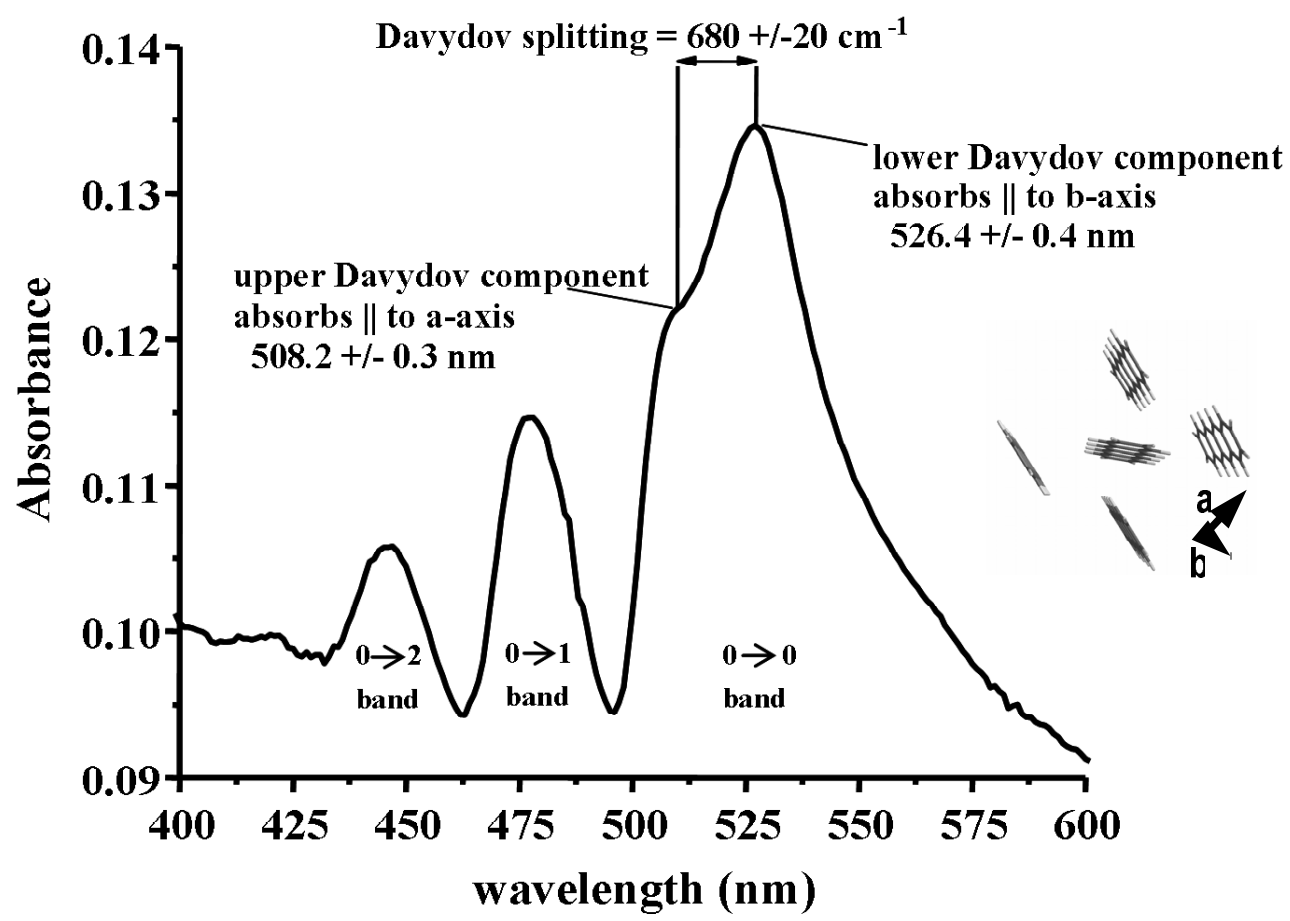

Fig. S3. Unpolarized UV-Vis absorption spectrum of tetracene film grown with PVA and LC 3417 on glass. This spectrum is virtually indistinguishable from those found in previous studies with regard to both peak positions and degree of Davydov splitting, a broadening of the 0-0 band due ultimately to the unit cell structure of tetracene which is comprised of two unequal molecules

Considering the lower Davydov component centered $\sim 527 \mathrm{~nm}$, the absorbance of a single, isolated crystal is:

$$
\mathrm{A}^{527}=\mathrm{A}_{\text {back }}^{527}+\mathrm{k}^{2} \cos ^{2}(\phi)
$$

where $A_{b a c k}^{527}$ is background absorbance and scattering, $k$ is an absorption parameter determined by crystal thickness and the molar extinction coefficient, and $\phi$ is the angle between the electric field vector and the $b$-axis. In a film containing many crystals, the overall absorbance profile reflects the macroscopic distribution of orientations. As we have shown, films grown using an oriented LC solvent exhibit two dominant crystalline orientations, related to one another other by a mirror plane along the nematic director. The films are highly ordered, so for simplicity the $b$-axis can be modeled as orienting equally often along just two in-plane directions, $\pm \beta$, where $\beta=0$ is defined parallel to the director. The analysis of just two orientations, rather than two ensembles of orientations, does not affect the conclusion. The overall absorbance is:

$$
A_{\text {overall }}^{527} \approx A_{\text {back }}^{527}+\operatorname{kcos}^{2}(\theta+\beta)+\operatorname{kcos}^{2}(\theta-\beta) \quad \text { (Eq. S2) }
$$


where $\theta$ is the angle between the electric field vector and the rubbing direction of the LC alignment layer.

As can be seen from the form of Equation S2, an analysis of the polarization dependent absorption of a macroscopic film is limited to revealing whether or not the $b$-axis orients within $\pm 45^{\circ}$ of the rubbing direction - no further orientational information can be deduced due to the film's mirror symmetry. Fitting Eq. S2 to polarized spectra from a tetracene film grown on glass using ZLI-3417 and a rubbed PVA alignment layer (Fig. S1) shows $\beta>45^{\circ}$, which agrees with the results from crystal shape modeling $\left(\beta=55.13^{\circ}\right)$, and is consistent with an assignment placing the [110] axis parallel to the nematic director.

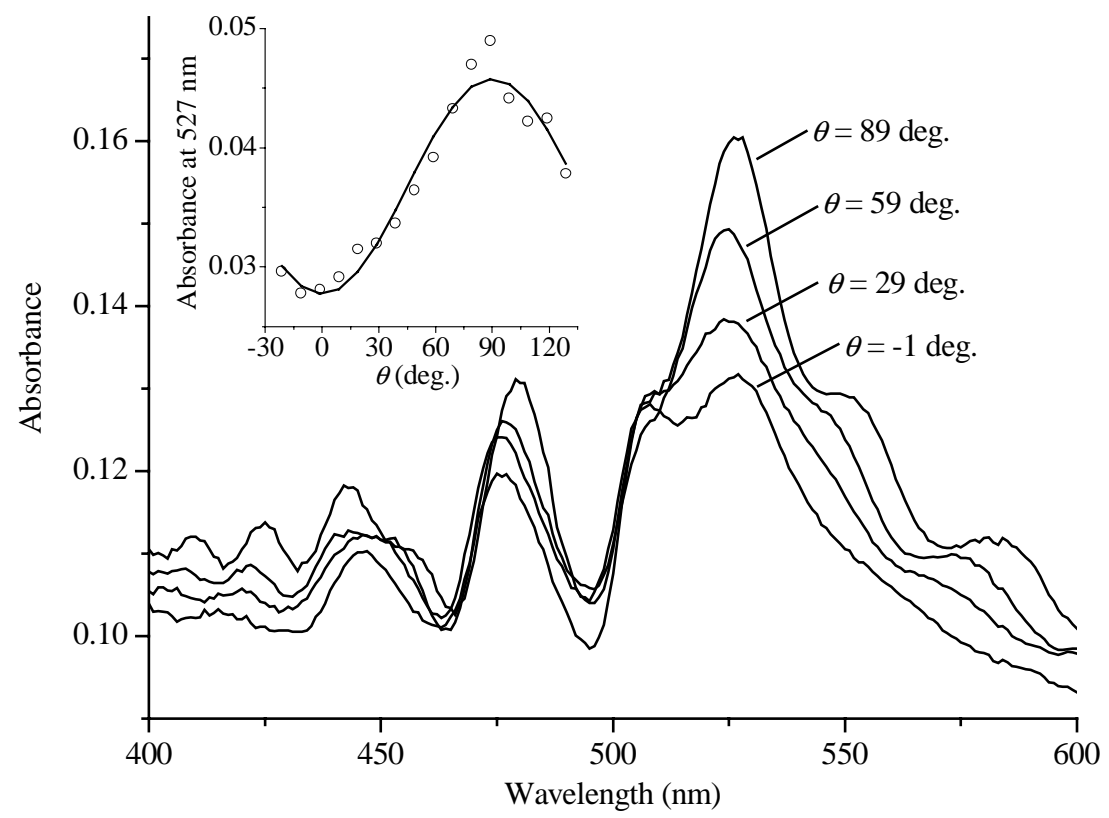

Fig. S4. Analysis of an oriented tetracene film on glass / rubbed PVA / ZLI-3417 using polarized UVVis absorption spectroscopy. The main figure shows spectra acquired at four different polarizer orientations, where the indicated angle was that between the nematic director and the electric field vector. The inset shows the absorbance of the lower Davydov component at $527 \mathrm{~nm}$, along with a fit using Eq. S2. 


\section{References}

1 Sondermann, U.; Bassler, H., J. Phys. Chem. 1985, 89, 1735.

2 "Spectroscopic Approach to Energetics of Exciton Fission and Fusion in Tetracene Crystals", Tomkiewicz, Y., Groff, R. P., and Avakian P., J. Chem. Phys., 54, 4504, (1971).

3 "Fluorescence of Solid Tetracene in the Temperature Range 4.2 to 300 K", Muller, H. and Bassler, H. Chem. Phys. Lett., 36, 312, (1975).

4 "Exciton Delocalization and Superradiance in Tetracene Thin Films and Nanoaggregates", Lim, S. H., Bjorklund, T.G., Spano, F.C., and Bardeen, C.J., Phys. Rev. Lett., 92, 107402, (2004).

5 "Intermediate Exiton-Phonon Coupling in Tetracene", Mizuno K., Matsui, A., and Sloan, G. J., J. Phys. Soc. Jpn, (1984), 53, 2799-2806.

6 "Diffusion of Singlet Excitons in Tetracene Crystals", Vaubel, G., Baessler, H., Mol. Cryst. Liq. Cryst., 12, 39, (1970).

7 "Pressure-Induced Phase Transition in Tetracene Crystals", Kalinowski, J., Godlewski, J., and Jankowiak, R., Chem. Phys. Lett., 43, 127, (1976).

8 "Pressure Dependence of the Davydov Splitting in Tetracene Single Crystals", Kalinowski, J. and R. Jankowiak, R., Chem. Phys. Lett. 53, 56, (1978).

9 "The Electronic Spectra of Pyrene, Chrysene, Azulene, Coronene and Tetracene Crystals", J. Tanaka, Bull. Chem. Soc. Jpn., 38, 86-103, (1965).

10 Sakurai, T.; Hayakawa, S., Jpn. J. Appl. Phys., 1974, 13, 1733; Mizuno, K.; Matsui, A.; Sloan, G. J., J. Phys. Soc. Jpn, 1984, 53, 2799; Tanaka, J., Bull. Chem. Soc. Jpn., 1965, 38, 86. 